\title{
THE EFFECT OF CHLORAMPHENICOL IN SALMONELLA ENTERITIS OF INFANCY
}

BY

\author{
W. B. MACDONALD, FREDA FRIDAY and MERRAN MCEACHARN \\ From the Department of Clinical Research and the Department of Pathology, Royal Children's Hospital, Melbourne
}

(RECEIVED FOR PUBLICATION DECEMBER 29, 1953)

During the year 1951, in the Royal Children's Hospital, Melbourne, chloramphenicol was used for the treatment of numerous children with infective diarrhoea, and an impression was gained that it was relatively ineffective in the treatment of Salmonella enteritis, despite the high sensitivity of the group to chloramphenicol in vitro. Experimental work on mice by Seligmann and Wassermann (1949) indicated that chloramphenicol was bacteriostatic rather than bacteriocidal to Salmonella. The clinical results of Ross, Burke, Rice, Washington and Stevens (1950), Weiner and Liebler (1951) and Kunstadter, Milzer and Kagan (1951) on small series of patients supported this finding, but the variable clinical course of Salmonella enteritis, even when untreated, made assessment of the efficacy of antibiotics difficult. In some cases the organism temporarily disappeared from stools during treatment, but reappeared soon after cessation of therapy. Fison and Singer (1950), in treating an outbreak of infantile diarrhoea due to Salmonella, formed the opinion that chloramphenicol was responsible for decreasing morbidity and mortality, and other reports of single cases or small series supported this view.

At the Royal Children's Hospital, Melbourne, from November, 1951, to March, 1953, a controlled clinical therapeutic trial to determine the efficacy of chloramphenicol on infants suffering from Salmonella enteritis was carried out. During this period, 390 infants less than 2 years of age were admitted to hospital with the diagnosis of infective diarrhoea. Salmonella were grown from the stools of 98 of these infants, 51 of whom were studied in the controlled trial.

\section{Methods}

As soon as an infant with diarrhoea had been admitted to hospital, two rectal swabs following the passage of stools were cultured immediately on 'difco' S.S. agar and in tetrathionate broth. From these two primary cultures subsequent isolation and identification were performed. If a culture of either specimen grew Salmonella, it was considered that the infant fulfilled the bacteriological requirements for admission to the controlled therapeutic trial.

In addition, all infants included in the trial were less than 2 years of age, were in good health before the onset of enteritis, and were free from parenteral infection. In each case, the enteritis was of less than one week's duration, and no specific treatment had been given before admission to hospital. A history of feeding derangement precluded admission to the series. Although this pre-selection reduced the number of infants studied to approximately half the cases of Salmonella enteritis admitted to hospital the validity of the results was improved by the etimination of variables. All-infants were moderately ill with eight to 20 bowel actions per day. Clinical dehydration severe enough to warrant intravenous infusion was the exception, and as preliminary observations had revealed no obvious relationship between initial dehydration and the duration of the illness, this physical feature played no part in pre-selection.

Treated and control infants were selected on the basis of 'odds and evens' taken from a table of random numbers. After it had been decided by the medical observer that an infant fulfilled the clinical and bacteriological requirements the ward sister allotted it to the treated or untreated group, but the category was not known to the medical observer. Thus, as there was no alternation of cases, observations were quite unbiased.

Treated infants received oral chloramphenicol, $120 \mathrm{mg}$. $/ \mathrm{kg}$. of body weight per day, at six- to eighthourly intervals for 10 days. Apart from chloramphenicol, treated and control infants received the same treatment. A similar feeding routine was established in each case, modified only by the course of the illness and the age of the infant. After an initial starvation of 24 hours, during which time water with $5 \%$ glucose and a solution of sodium and potassium salts were given, the feeding was changed 
TABLE 1

AGE GROUPING OF 25 INFANTS TREATED WITH CHLORAMPHENICOL AND 26 CONTROLS

\begin{tabular}{|c|c|c|c|c|c|c|}
\hline Category & & Less than 3 months & 3-6 months & 6-12 months & 12-18 months & 18-24 months \\
\hline Treated & $\cdots$ & 4 & 5 & 13 & 3 & Nil \\
\hline Controls & $\cdots$ & 4 & 8 & 9 & 3 & 2 \\
\hline
\end{tabular}

to half-strength skimmed milk, thence to fullstrength skimmed milk, and finally, to whole dried milk, occasionally through an intermediate stage of half-cream dried milk, with formula according to age. Symptomatic treatment was confined to sedation, and topical applications to rashes and excoriations. Although Salmonella enteritis had a higher incidence during the summer months, infants suffering from this disease were admitted to hospital throughout the winter. To avert complicating respiratory infections, penicillin was sometimes given to untreated infants, and although this introduced another variable, it was considered to be less confusing than a complicating respiratory infection of which only the mildest were encountered. Ordering penicillin for untreated cases with respiratory infections was the responsibility of the ward registrar.

For 10 days following isolation of Salmonella stools were cultured on every second day from both treated and control infants, and, after the conclusion of treatment, on every third day until discharge from hospital. The infants were reviewed one week later in the out-patient department when a rectal swab was cultured.

\section{Results}

Of a total of 98 infants with Salmonella infection, 51 were included in the experiment. Of these 51 infants, 48 were infected by $S$. typhi-murium, two by $S$. adelaide, and one by $S$. derby. Twenty-five of the 51 infants were treated, and 26 acted as controls, a distribution as good as if treatment had been allotted to alternate cases. The 25 treated infants consisted of 15 males and 10 females, and 14 males and 12 females comprised the untreated group of 26 infants. The infecting organisms in the treated group were $S$. typhi-murium (23 cases), $S$. adelaide (one case) and $S$. derby (one case), and in the untreated group, $S$. typhi-murium ( 25 cases) and $S$. adelaide (one case). Age grouping was reasonably well distributed (Table 1). The mean age of the treated infants was $7 \cdot 44$ months, the range being from 1 to 17 months, while the mean age of the untreated group was 8.07 months, with a range of 2 to 21 months. The effect of chloramphenicol was assessed both clinically and bacteriologically.

\section{Clinical Assessment}

It was considered that a clinical cure had been obtained when an infant was tolerating a fullstrength feed, and was excreting stools of normal consistency and number for that particular age group. This decision was arbitrary and was dependent on the clinical experience of the medical observer. By the time this stage had been reached the infant was invariably afebrile and free from other abnormal features. Weight gain had usually begun but was not considered essential as an index of clinical cure, as a small infant after a protracted illness sometimes would take several weeks before he had gained satisfactorily. On this basis, clinical results of chloramphenicol are shown in Tables $2 a$ and $2 b$. The mean duration in days from the beginning of the illness to clinical cure was $19 \cdot 52$

TABLE 2a

MEAN DURATION AND RANGE IN DAYS FROM START OF ILLNESS TO CLINICAL CURE

\begin{tabular}{cc|c|c|c|c|c|c|c|}
\hline \multicolumn{1}{c|}{ Category } & & Less than 3 months & $3-6$ months & 6-12 months & $12-18$ months \\
\hline Treated & $\ldots$ & $25 \cdot 75(8-46)$ & $18 \cdot 4(12-24)$ & $19 \cdot 3(10-35)$ & $14 \cdot 0(12-16)$ \\
Controls & $\cdots$ & $24 \cdot 25(19-25)$ & $21 \cdot 1(12-38)$ & $20 \cdot 4(10-38)$ & $12 \cdot 6(3-18)$ \\
\hline
\end{tabular}

TABLE $2 \mathrm{~b}$

MEAN DURATION AND RANGE IN DAYS FROM START OF TREATMENT TO CLINICAL CURE

\begin{tabular}{|c|c|c|c|c|c|c|}
\hline Category & & Less than 3 months & 3-6 months & 6-12 months & 12-18 months & 18-24 months \\
\hline Treated & $\cdots$ & $21 \cdot 75(6-41)$ & $12 \cdot 2(9-16)$ & $14 \cdot 8(7-32)$ & $10 \cdot 3(7-12)$ & Nil \\
\hline Controls & .. & $18 \cdot 5(11-30)$ & $15 \cdot 9(9-33)$ & $13 \cdot 75(3-30)$ & $7 \cdot 3(0-15)$ & $8 \cdot 5(8-9)$ \\
\hline
\end{tabular}


days, ranging from eight to 46 days (standard deviation $9 \cdot 24$ days) for treated infants and 18.96 days ranging from three to 38 days (standard deviation $9 \cdot 10$ days) for control infants. The mean duration in days from the beginning of treatment to clinical cure was 14.90 days, ranging from six to 41 days (standard deviation 9.09 days) for treated infants, and 13.46 days, ranging from nil to 33 days (standard deviation $8 \cdot 27$ days) for control infants.

In Tables $2 a$ and $2 b$ the mean duration and range in days have been tabulated according to age. There appears to be an inverse relationship of age to length of illness, but the number of cases in each age category is too small and the range too large to be significant. Three infants only required intravenous infusion; two were in the control group and one was treated. The two untreated infants were clinically cured in 10 and 36 days from the beginning of their illnesses, and in seven and 33 days from when treatment would have begun. The treated infant was clinically cured in 19 days from the beginning of his illness and in 18 days from the start of treatment. The treated infant infected with $S$. adelaide was clinically cured in 18 days after the start of illness, and in 16 days following the beginning of treatment. The treated infant infected with $S$. derby was clinically cured in 17 days after the beginning of his illness, and in 13 days after the start of treatment. The control infant infected with $S$. adelaide was cured in 11 days after his illness started and in nine days from when treatment would have been begun. It appears that the clinical course of infection with these two strains of Salmonella is similar to that with $S$. typhi-murium, as far as one can determine from three cases.

\section{Bacteriological Assessment}

The Salmonella group was moderately sensitive to chloramphenicol in vitro, and most organisms tested were sensitive to a concentration of $5 \mu \mathrm{g} . / \mathrm{ml}$. A few were resistant to $5 \mu \mathrm{g}$. $/ \mathrm{ml}$. but were sensitive to $10 \mu \mathrm{g} . / \mathrm{ml}$. The effect in vivo was completely unpredictable both in treated and untreated infants. Isolation of Salmonella was obtained at various stages of the clinical course, with comparable frequency in both treated and untreated cases. The results have been categorized arbitrarily in Table 3 . At the time of discharge from hospital, Salmonella could still be cultured from the stools of 10 treated infants and 11 untreated infants. In four treated infants and in three controls, the organism had temporarily disappeared during the stay in hospital, but was present on discharge. Forty-three of the 51 infants, comprising 22 treated and 21 control cases, were examined in the out-patient department one to two weeks after discharge from hospital, and rectal swabbings were cultured from each. Salmonella was isolated from eight treated and eight control infants. The behaviour of Salmonella was unpredictable in the control infants as well as in those treated with chloramphenicol, and on these figures even a temporary bacteriostatic effect of the drug in vivo could not be claimed, as in 15 untreated, as well as in 15 treated cases, the organism could not be demonstrated on stool culture at the time of discharge from hospital.

\section{Discussion}

Although minor epidemics may occur during summer, $S$. typhi-murium appears to be endemic in Melbourne, and infants suffering from Salmonella enteritis are admitted to hospital each week. However, during a period of 17 months with the preselection used in the present procedure, only 51 infants less than 2 years of age were found to be suitable for a controlled therapeutic trial of chloramphenicol. Restriction to a narrower group was thus not justified, despite a tendency for the clinical course of the disease to be protracted in the early months of life. The wide range in duration of the natural course of the disease diminishes the significance of results, but, as it was impossible to predict the duration or severity of the illness at its start, a further pre-selection to demonstrate the efficacy of chloramphenicol better was impracticable. Despite these incompletely controlled variables, results at least demonstrate that in the practical management of Salmonella enteritis in infants administration of

TABLE 3

TIME OF DISAPPEARANCE OF SALMONELLA FROM STOOL CULTURES RELATIVE TO TREATMENT OR WHEN TREATMENT WOULD HAVE BEEN GIVEN TO CONTROLS

\begin{tabular}{|c|c|c|c|c|c|}
\hline Category & $\begin{array}{l}\text { Isolated on 1st pre- } \\
\text { treatment Stool } \\
\text { Culture but not } \\
\text { Subsequently }\end{array}$ & $\begin{array}{l}\text { Disappearance during } \\
\text { Treatment without } \\
\text { Reappearance }\end{array}$ & $\begin{array}{l}\text { Disappearance during } \\
\text { Treatment with } \\
\text { Recurrence during or } \\
\text { after Treatment }\end{array}$ & $\begin{array}{c}\text { Disappearance after } \\
\text { Treatment before } \\
\text { Discharge from } \\
\text { Hospital }\end{array}$ & $\begin{array}{l}\text { Present throughout } \\
\text { Stay in Hospital } \\
\text { and on Discharge }\end{array}$ \\
\hline Treated $\quad$. & 2 & 9 & 8 & Nil & 6 \\
\hline Controls ... & 1 & 7 & 7 & 3 & 8 \\
\hline
\end{tabular}


chloramphenicol in adequate dosage has no effect in altering the clinical course of the disease, or of significantly affecting the growth of the organism in the stools. There was no evidence that the drug adversely affected the course of the disease, but continuation of its use would appear unnecessary and uneconomical. Serum concentrations of chloramphenicol were not estimated in this trial. The sensitivity of the Salmonella group in vitro was of the same order as that demonstrated by McLean, Schwab, Hillegas and Schlingman (1949) and by other workers since then.

\section{Summary}

Fifty-one infants less than 2 years old suffering from Salmonella enteritis were subjected to a controlled therapeutic trial to test the efficacy of chloramphenicol in this disease.

Forty-eight of the infants were infected by S. typhi-murium.

Chloramphenicol at a dose of $120 \mathrm{mg} . / \mathrm{kg} . /$ day had no effect in influencing the rate of clinical recovery.

Despite a high degree of sensitivity to chloramphenicol in vitro, the growth of Salmonella in the bowel was not significantly affected by the drug.

Doctors D. H. McCredie, M. Clare McKinnon, J. J. M. O'Neill, and M. J. Robinson, of the resident medical staff, Royal Children's Hospital, assisted in the practical management and observations of the infants.

Sisters J. R. Scott, M. M. McQuarrie, and M. Houston were responsible for the nursing care and observations, and the allotment of infants to the treated or control groups.

\section{REFERENCES}

Fison, D. C. and Singer, E. (1950). Med. J. Aust., $2,957$. Kunstadter, R. H., Milzer, A. and Kagan, B. M. (1951). J. Pediat.,

McLean, I. W. Jr., Schwab, J. L., Hillegas, A. B. and Schlingman, A. S. (1949). J. clin. Invest., 28, 953.

Ross, S. Burke F. G Rice E. C. Washington, J. A. and Stevens, S. (i950). New Engl. J. Med., $242,173$.

Seligmann, E. and Wassermann, M. (1949). Proc. Soc. exp. Biol. $N . Y ., 71,253$.

Weiner, H.' A. and Liebler, J. B. (1951). J. Amer. med. Ass., 145, 802 